\title{
On the Instability of the BDF Formulas
}

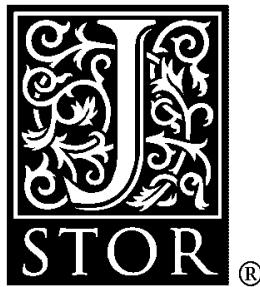

\author{
E. Hairer; G. Wanner
}

SIAM Journal on Numerical Analysis, Vol. 20, No. 6. (Dec., 1983), pp. 1206-1209.

Stable URL:

http://links.jstor.org/sici?sici=0036-1429\%28198312\%2920\%3A6\%3C1206\%3AOTIOTB\%3E2.0.CO\%3B2-2

SIAM Journal on Numerical Analysis is currently published by Society for Industrial and Applied Mathematics.

Your use of the JSTOR archive indicates your acceptance of JSTOR's Terms and Conditions of Use, available at

http://www.jstor.org/about/terms.html. JSTOR's Terms and Conditions of Use provides, in part, that unless you have obtained prior permission, you may not download an entire issue of a journal or multiple copies of articles, and you may use content in the JSTOR archive only for your personal, non-commercial use.

Please contact the publisher regarding any further use of this work. Publisher contact information may be obtained at http://www.jstor.org/journals/siam.html.

Each copy of any part of a JSTOR transmission must contain the same copyright notice that appears on the screen or printed page of such transmission.

JSTOR is an independent not-for-profit organization dedicated to and preserving a digital archive of scholarly journals. For more information regarding JSTOR, please contact support@jstor.org. 


\title{
ON THE INSTABILITY OF THE BDF FORMULAS*
}

\author{
E. HAIRER $\dagger$ AND G. WANNER $\ddagger$
}

Dedicated to Peter Henrici on the occasion of his 60th birthday

Abstract. We give a short proof of the instability of the BDF formulas for $k \geqq 7$.

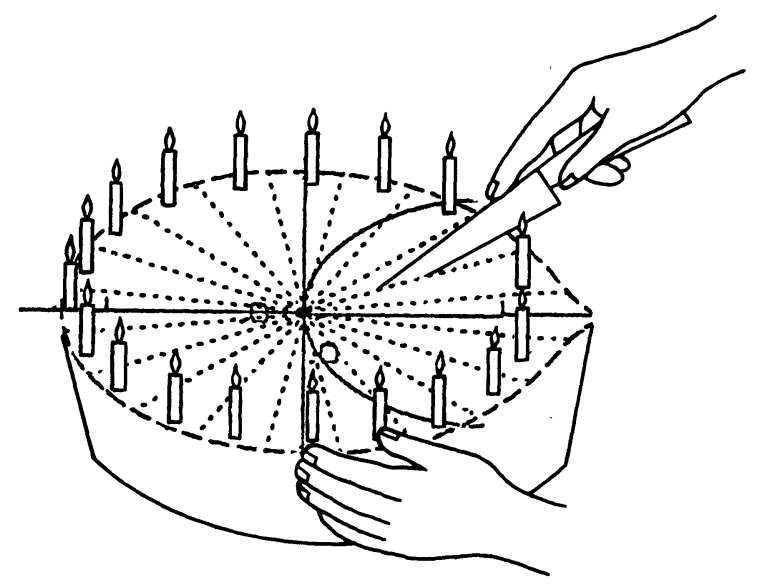

The backward difference formulas

$$
\sum_{j=1}^{k} \frac{1}{j} \nabla^{j} y_{n+1}=h \dot{f_{n+1}}
$$

are the most widely used methods for the solution of (stiff) differential equations and became particularly prominent with the books of Henrici (1962) and Gear (1971). For 30 years it has been "known" from numerical calculations that they are unstable for $k \geqq 7$ (Mitchell and Craggs (1953)), but a rigorous mathematical proof of this fact has only been given as late as 1971 in a fifty-page report of Cryer (see Cryer (1971), (1972), also Creedon and Miller (1975)).

THEOREM. The $k$-step BDF formula (1) is stable for $k \leqq 6$, and unstable for $k \geqq 7$.

Proof. As the $\rho$-polynomial of (1) is given by

$$
\rho(\zeta)=\sum_{j=1}^{k} \frac{1}{j} \zeta^{k-j}(\zeta-1)^{j}
$$

the transformation $\zeta=1 /(1-z)$ yields

$$
p(z)=(1-z)^{k} \rho(\zeta)=\sum_{j=1}^{k} \frac{z^{j}}{j} .
$$

* Received by the editors July 13, 1982, and in revised form March 15, 1983.

† Universität Heidelberg, Institut für Angwandte Mathematik, Im Neuenheimer Feld 294, D-69 Heidelberg 1, West Germany.

¥Section de Mathématiques, Université de Genève, Case Postale 240, CH-1211 Genève 24, Switzerland. 
Hence, by definition of stability, formula (1) is stable iff all roots of (2) are outside the disk $\{z ;|z-1| \leqq 1\}$, with simple roots allowed on the boundary.

It is known (see Jentzsch $(1918$, p. 219)) that each point on the circle of convergence of a Taylor series is a limit point of the zeros of the truncated series (2); therefore instability is necessary for $k$ tending to infinity. However, for $k$ fixed, we need a more refined estimation.

The roots of (2) are displayed in Fig. 1 for different values of $k$, and the validity of the theorem can be observed for $k \leqq 11$. For $k$ larger, we write

$$
p(z)=\int_{0}^{z} \sum_{j=1}^{k} \zeta^{j-1} d \zeta=\int_{0}^{z} \frac{1-\zeta^{k}}{1-\zeta} d \zeta=\int_{0}^{r}\left(1-e^{i k \theta} s^{k}\right) \phi(s) d s
$$

with

$$
\zeta=s e^{i \theta}, \quad z=r e^{i \theta}, \quad \phi(s)=\frac{e^{i \theta}}{1-s e^{i \theta}}
$$

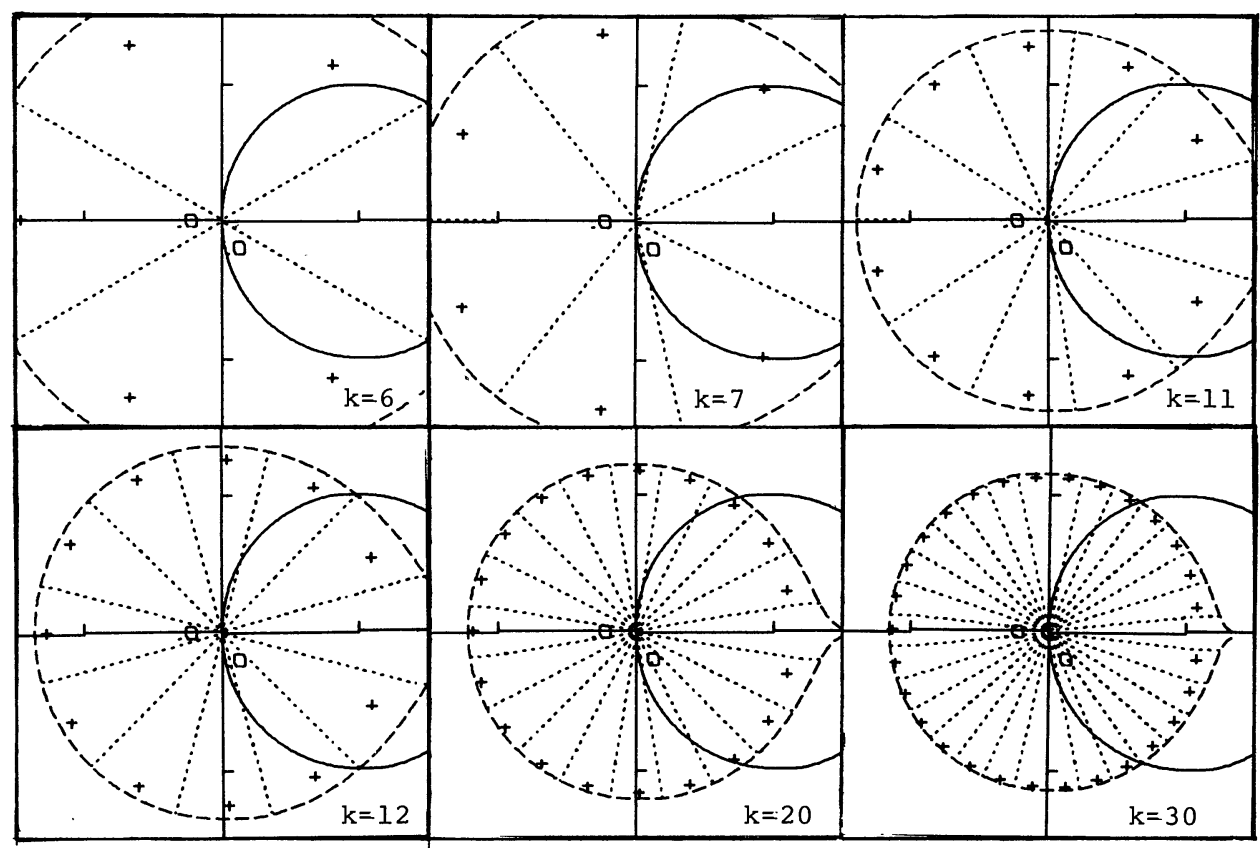

FIG. 1. Henrici's birthday cake $(+\cdots$ zeros of $p(z))$.

1. We cut the complex plane into $k$ sectors,

$$
\begin{aligned}
S_{j}=\left\{z ; \frac{2 \pi\left(j-\frac{1}{2}\right)}{k}<\arg (z)<\frac{2 \pi\left(j+\frac{1}{2}\right)}{k}, \delta<|z|<R\right\}, \\
\quad j=0,1, \cdots, k-1, \delta \text { small, } R \text { large. }
\end{aligned}
$$

On the rays limiting $S_{j}$ we have $e^{i k \theta}=-1$, so that from (3)

$$
p(z)=\int_{0}^{r}\left(1+s^{k}\right) \phi(s) d s
$$

with a positive weight function. Therefore the argument of $p(z)$ lies always in the angle which is spanned by $\phi(s)$, i.e., the angle between $e^{i \theta}$ and $e^{i \pi}=-1$. So no 
revolution of $\arg (p(z))$ is possible on these rays, and, due to the one revolution of $\arg \left(z^{k}\right)$ at infinity between $\theta=2 \pi\left(j-\frac{1}{2}\right) / k$ and $\theta=2 \pi\left(j+\frac{1}{2}\right) / k$, the principle of the argument (e.g. Henrici (1974, p. 278)) implies (see Fig. 2):

In each sector $S_{j}(j=1, \cdots, k-1$, with the exception of $j=0)$ lies exactly one root of $p(z)$.

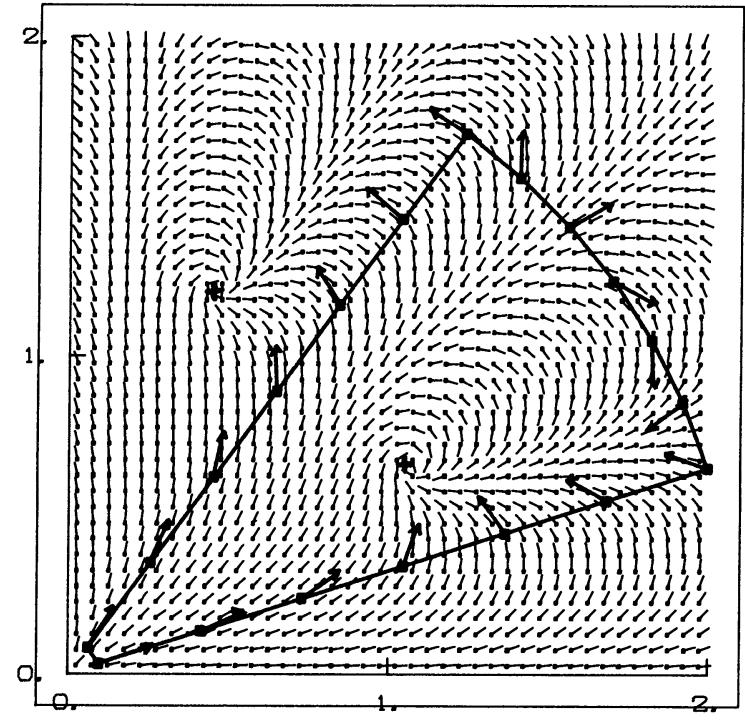

FIG. 2. The first sector $S_{1}$ for $k=10$ with indication of $\arg (p(z))$.

2. In order to complete the proof, we will have to bound the zeros of $p(z)$ from above. We observe that in (3) the term $s^{k}$ becomes large for $s>1$. We therefore partition (3) into two integrals $p(z)=I_{1}-I_{2}$, where

$$
I_{1}=\int_{0}^{r} \phi(s) d s-\int_{0}^{1} e^{i k \theta} s^{k} \phi(s) d s, \quad I_{2}=e^{i \theta k} \int_{1}^{r} s^{k} \phi(s) d s .
$$

Since $|\phi(s)| \leqq B(\theta)$, where

$$
B(\theta)= \begin{cases}\frac{1}{\sin \theta}, & 0<\theta \leqq \frac{\pi}{2}, \\ 1, & \frac{\pi}{2} \leqq \theta \leqq \pi,\end{cases}
$$

we obtain

$$
\left|I_{1}\right| \leqq\left(r+\frac{1}{k+1}\right) B(\theta)<r B(\theta) \frac{k+2}{k+1} \quad(r>1) .
$$

Secondly, since $s^{k}$ is positive,

$$
I_{2}=e^{i \theta k} \phi \int_{1}^{r} s^{k} d s, \quad \text { where } \phi \in \operatorname{conv}\{\phi(s) ; 1 \leqq s \leqq r\} .
$$

Since by some elementary geometry we have $|\phi| \geq 1 / 2 r$ for $r \geqq 1$, and get

$$
\left|I_{2}\right| \geqq \frac{r^{k+1}-1}{k+1} \frac{1}{2 r}>r \frac{r^{k-1}-1}{2 k+2} \quad(r>1) .
$$


From (5) and (6) we obtain that

$$
r \geqq R(\theta)=((2 k+4) B(\theta)+1)^{1 /(k-1)}
$$

implies $\left|I_{2}\right|>\left|I_{1}\right|$, so that $p(z)$ cannot be zero. The curve $R(\theta)$ is also plotted in Fig. 1 and cuts from the sectors $S_{j}$, what we call "Henrici's birthday cake," each slice of which (with $j \neq 0$ ) must contain a zero of $p(z)$. A simple analysis shows that for $k=12$ the cake, for $S_{1}$, is small enough to assure the presence of zeros of $p(z)$ inside the disk $\{z ;|z-1| \leqq 1\}$. As $R(\theta)$, for fixed $\theta$, as well as $R(\pi / k)$ are monotonically decreasing in $k$, the same is true for all $k \geqq 12$.

Acknowledgment. The authors wish to thank Prof. R. Jeltsch for helpful comments and references.

\section{REFERENCES}

D. M. CReEdon AND J. J. H. Miller (1975), The stability properties of q-step backward difference schemes, BIT, 15, pp. 244-249.

C. W. CRYER (1971), A proof of the instability of backward-difference multistep methods for the numerical integration of ordinary differential equations, Tech. Rep. 117, Computer Science Dept., Univ. Wisconsin, Madison, pp. 1-52.

, (1972), On the instability of high order backward-difference multistep methods, BIT 12, pp. 17-25.

C. W. Gear (1971), Numerical Initial Value Problems in Ordinary Differential Equations, Prentice-Hall, Englewood Cliffs, NJ.

P. Henrici (1962), Discrete Variable Methods in Ordinary Differential Equations, John Wiley, New York. , (1974), Applied and Computational Complex Analysis I, John Wiley, New York.

A. R. Mitchell AND J. W. CRAGGS (1953), Stability of difference relations in the solution of ordinary differential equations, MTAC 7, pp. 127-129.

R. JENTZSCH (1918), Untersuchungen zur Theorie der Folgen analytischer Funktionen, Acta Math., 41, pp. 219-251. 